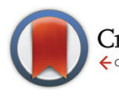

CrossMark

\&click for updates

Cite this: Polym. Chem., 2016, 7, 2955

Received 21st February 2016,

Accepted 1st April 2016

DOI: 10.1039/c6py00325g

www.rsc.org/polymers

\title{
Tailoring the structure of polymer networks with iniferter-mediated photo-growth
}

\author{
Awaneesh Singh, ${ }^{a}$ Olga Kuksenok, ${ }^{b}$ Jeremiah A. Johnson ${ }^{\star c}$ and Anna C. Balazs ${ }^{\star a}$ \\ Using dissipative particle dynamics (DPD), we developed a computational approach to capture the photo- \\ controlled radical polymerization ("photo-growth") of polymer gels containing trithiocarbonate (TTC) \\ groups within the network strands. Using this model, we focused on a "primary gel" and illuminated the \\ sample to activate the TTCs, which then interacted with monomer and cross-linker in the solution. At low \\ TTC concentrations, gels composed of compatible monomers formed two distinct, spatially separated \\ layers. Conversely, at high TTC concentration, gels formed from incompatible components displayed a \\ well-intermixed structure. Hence, in the presence of light, variations in the TTC concentration provide a \\ new approach for controllably tailoring the structure of polymer gels, and thereby tailoring the functional- \\ ity of the network.
}

\section{Introduction}

The development of new strategies to impart externally switchable behavior to gels will guide the design and application of the next-generation of stimuli-responsive soft materials. Light is a particularly convenient source of energy for altering and/ or switching gel properties, and there are now many examples of gels that degrade, bend, heal, etc. in response to light. ${ }^{1-16}$ Furthermore, the use of light to initiate controlled polymerization has witnessed an explosion of interest in recent years $;{ }^{17-25}$ there are now variants of many of the most wellknown controlled radical polymerization (CRP) reactions that employ light to switch chain propagation between "on" and "off" states. ${ }^{26-28}$

In 2013, Johnson et al. ${ }^{29}$ reported a strategy for altering the structure and composition of covalent polymer gels by direct, living extension of the network chains via photo-controlled radical polymerization (photo-CRP). This process, which was called "photo-growth", was facilitated by the use of UV-lightresponsive trithiocarbonate (TTC) "iniferters" embedded within the gel network. The term "iniferter" was introduced by Otsu et al. ${ }^{30}$ to define a compound that serves as an initiator, chain-transfer agent, and terminator (hence the name inifer-ter) in controlled free radical polymerizations. ${ }^{30,31}$ When the photo-responsive TTC iniferter is exposed to light, it dis-

\footnotetext{
${ }^{a}$ Chemical Engineering Department, University of Pittsburgh, Pittsburgh, PA 15261, USA.E-mail: balazs@pitt.edu

${ }^{b}$ Materials Science and Engineering Department, Clemson University, Clemson, SC 29634, USA

${ }^{c}$ Chemistry Department, Massachusetts Institute of Technology, Cambridge, MA 02139, USA. E-mail:jaj2109@mit.edu
}

sociates into two fragments: a carbon-centered radical that can initiate polymerization and a TTC-based radical that can reversibly terminate growing chains. Hence, irradiation of the solvent-swollen gel in the presence of monomer led to transient chain cleavage and living photo-CRP; removal of the light restored the network structure via reversible radical deactivation. Thus, the molecular weight between junctions increased in response to light, which led to bulk network growth (increased swelling). The photo-growth strategy offers a unique way to externally tune both the mechanical and chemical properties of gels using light along with various monomers and cross-linkers. ${ }^{16}$ Furthermore, recent advances in the use of TTCs for photo-CRP, in particular the development of photoredox catalyzed methods that allow polymerization under visible and even near infrared light, ${ }^{28,32-35}$ could in principle be applied within the photo-growth paradigm to yield novel smart materials. Thus, we seek to gain a deeper understanding of the unique features of photo-growth within polymer gels.

Computer simulations can play a particularly useful role in enhancing our understanding of such polymerization reactions and gel formation, allowing us to characterize important features such as the distribution of reactive chain-ends, the evolution of the molecular weight distributions and the structure of the polymer matrix. We recently developed the first computational approach based on the dissipative particle dynamics (DPD) method to simulate the living copolymerization of monomer and cross-linker to form a polymer network. ${ }^{36,37}$ Our model captured the characteristic polymerization kinetics of atom transfer radical polymerization $(\text { ATRP })^{38-41}$ and reproduced features of gel formation that are consistent with prior experimental data. ${ }^{36}$ We also developed a new DPD model to simulate free radical polymerization 
(FRP), ${ }^{42}$ taking into account different mechanisms of chain termination. The latter simulations allowed us to systematically examine the relative effects of the initiation, propagation, and termination reactions in the formation of polymer-clay nanocomposite gels, and hence, obtain greater insight into the polymerization process.

Here, we adapt our recently developed DPD framework to develop the first simulation approach for modeling TTC-based photo-CRP within polymer networks. In particular, we start with a "primary gel" and illuminate the sample to activate the TTCs, which then interact with monomer and cross-linker in the solution. By varying the TTC concentration within the primary gel, we establish a route for forming a spatially intermixed, hybrid gel from chemically incompatible monomers. We also isolate parameters where phase-separated gels are formed from chemically compatible monomers. Hence, in the presence of light, variations in the TTC concentration provide a new approach for controllably tailoring the structure of polymer gels, and adapting these materials for a range of different functions.

\section{Computational method}

Dissipative particle dynamics (DPD) $)^{43-45}$ provides a powerful computational approach for integrating both the reaction kinetics of polymerization processes and the dynamic behavior of the evolving complex fluid. ${ }^{36,37,42}$ More generally, DPD can be viewed as a coarse-grained molecular dynamics (MD) approach. Namely, the beads in DPD represent molecules (or clusters of molecules) rather than atoms and these molecules interact through a soft-core potential rather than the hard-core LennardJones potential typically used in MD. Hence, DPD allows one to capture phenomena on longer time and length scales than can be accessed through conventional MD simulations.

Similar to MD, DPD involves the numerical integration of Newton's equation of motion: $m \mathrm{~d} \mathbf{v}_{i} / \mathrm{d} t=\mathbf{f}_{i}$. Each bead $i$ experiences a force $\mathbf{f}_{i}(t)$ that is the sum of three pairwise additive forces $\mathbf{f}_{i}(t)=\sum\left(\mathbf{F}_{i j}^{\mathrm{C}}+\mathbf{F}_{i j}^{\mathrm{D}}+\mathbf{F}_{i j}^{\mathrm{R}}\right)$, where the sum runs over all beads $j$ within a certain cutoff radius $r_{\mathrm{c}}$. The conservative force is a soft, repulsive force given by $\mathbf{F}_{i j}^{\mathrm{C}}=a_{i j}\left(1-r_{i j}\right) \hat{\mathbf{r}}_{i j}$, where $a_{i j}$ is the maximum repulsion between beads $i$ and $j, r_{i j}=\left|\mathbf{r}_{i}-\mathbf{r}_{j}\right| / r_{\mathrm{c}}$ and $\hat{\mathbf{r}}_{i j}=\mathbf{r}_{i j} /\left|\mathbf{r}_{i j}\right|$. The soft-core force permits a degree of overlap between neighboring beads and the use of larger time steps than those normally employed in MD.

The drag force is $\mathbf{F}_{i j}^{\mathrm{D}}=-\gamma \omega_{\mathrm{D}}\left(r_{i j}\right)\left(\hat{\mathbf{r}}_{i j} \cdot \mathbf{v}_{i j}\right) \hat{\mathbf{r}}_{i j}$, where $\gamma$ is a simulation parameter related to the viscosity arising from the interactions between the beads (polymer-polymer, polymersolvent, and solvent-solvent), $\omega_{\mathrm{D}}$ is a weight function that goes to zero at $r_{\mathrm{c}}$, and $\mathbf{v}_{i j}=\mathbf{v}_{i}-\mathbf{v}_{j}$. The random force is $\mathbf{F}_{i j}^{\mathrm{R}}=$ $\sigma \omega_{\mathrm{R}}\left(r_{i j}\right) \xi_{i j} \hat{\mathbf{r}}_{i j}$, where $\xi_{i j}$ is a zero-mean Gaussian random variable of unit variance and $\sigma^{2}=2 \gamma k_{\mathrm{B}} T$. The value of $\gamma$ is chosen to ensure relatively rapid equilibration of the temperature in the system and the numerical stability of the simulations for the specified time-step. ${ }^{44}$ Here, $k_{\mathrm{B}}$ is the Boltzmann constant and $T$ is the temperature of the system. We select weight functions of the following form: $\omega_{\mathrm{D}}\left(r_{i j}\right)=\omega_{\mathrm{R}}\left(r_{i j}\right)^{2}=\left(1-r_{i j}\right)^{2}$ for $r_{i j}<r_{\mathrm{c} \cdot}{ }^{44}$ All three of these forces conserve momentum locally and therefore, hydrodynamic behavior emerges even in systems containing only a few hundred of particles. ${ }^{43-45}$

The equation of motion is integrated via a modified velocity-Verlet algorithm. ${ }^{46}$ In our simulation, we took $r_{\mathrm{c}}$ and $k_{\mathrm{B}} T$ as the characteristic length and energy scales, respectively. We chose the dimensionless value of $r_{\mathrm{c}}=1$ and room temperature as the reference value, setting $k_{\mathrm{B}} T_{0}=1$, where $T_{0}=298.15 \mathrm{~K}$. The reduced temperature is therefore defined as $T^{*}=T / T_{0}$. The characteristic time scale is then defined as $\tau=\left(m r_{\mathrm{c}}{ }^{2} / k_{\mathrm{B}} T_{0}\right)^{1 / 2}$. The remaining simulation parameters ${ }^{2}$ are $\gamma=4.5$ and $\Delta t=$ $0.02 \tau$, with a total bead number density of $\rho=3$.

The reactive components in the photo-growth process described herein are: TTCs, which serve as photo-initiators and reversible terminators, $N$-isopropylacrylamide (NIPAAm) monomers, and diacrylate, which constitutes a bifunctional cross-linker. In our simulations, all these components are modeled as DPD beads. The bifunctional cross-linker encompasses two reactive cross-linking units, but is modeled by one DPD bead with different "states", which indicate the effective reactivity of the cross-linker (i.e., the extent to which it has reacted). ${ }^{36,37}$

Given these components, we adapt our prior DPD approach for $\operatorname{ATRP}^{36,37}$ to model iniferter polymerization. First, we model the excitation of a TTC with light. ${ }^{29}$ Illumination of a TTC leads to the formation of two radicals via the fragmentation of the TTC group. As is typical in iniferter polymerizations, ${ }^{47}$ one reactive radical participates in initiation and then propagation; we refer to this fragment as the "active initiator". The other (less reactive) radical principally contributes to termination; we refer to this fragment as the "active TTC". Second, we introduce a scheme to simulate the termination of the propagating polymer chain in the absence of light to generate a polymeric TTC. ${ }^{29,32}$

The relevant steps in our DPD photo-CRP are shown schematically in Fig. 1a, indicating the initiation, propagation, cross-linking, and termination of growing chains. The asterisks in Fig. 1 represent the active radicals and the solid colors indicate the specific units in the scheme: inactive TTC (red), active TTC (orange), active initiator (cyan), monomers (green and blue), and the cross-linker (purple). (It is equally likely that the orange bead is on the left and the cyan is on the right, i.e., that the TTC is photo-cleaved on either side; here, we just show one of these two possible configurations.) As noted above, the orange radical (active TTC) cannot propagate, but it can undergo a chain termination reaction with the cyan radical. This termination reaction regenerates the inactive TTC, which can be initiated again by photocleavage. The open circles represent unreacted species and filled circles indicate fully reacted species. Note that in the proposed reaction scheme, we neglect degenerative chain transfer, decomposition of active TTCs and coupling of active TTCs to dormant TTCs $^{48}$ that prevents irreversible termination of the active ends. (In our simulations, the samples are continuously irradiated until complete polymerization has occurred; therefore, only a few unreacted TTCs are available in the system to par- 
(a) Initiation:

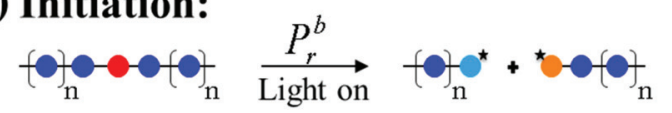

Propagation:
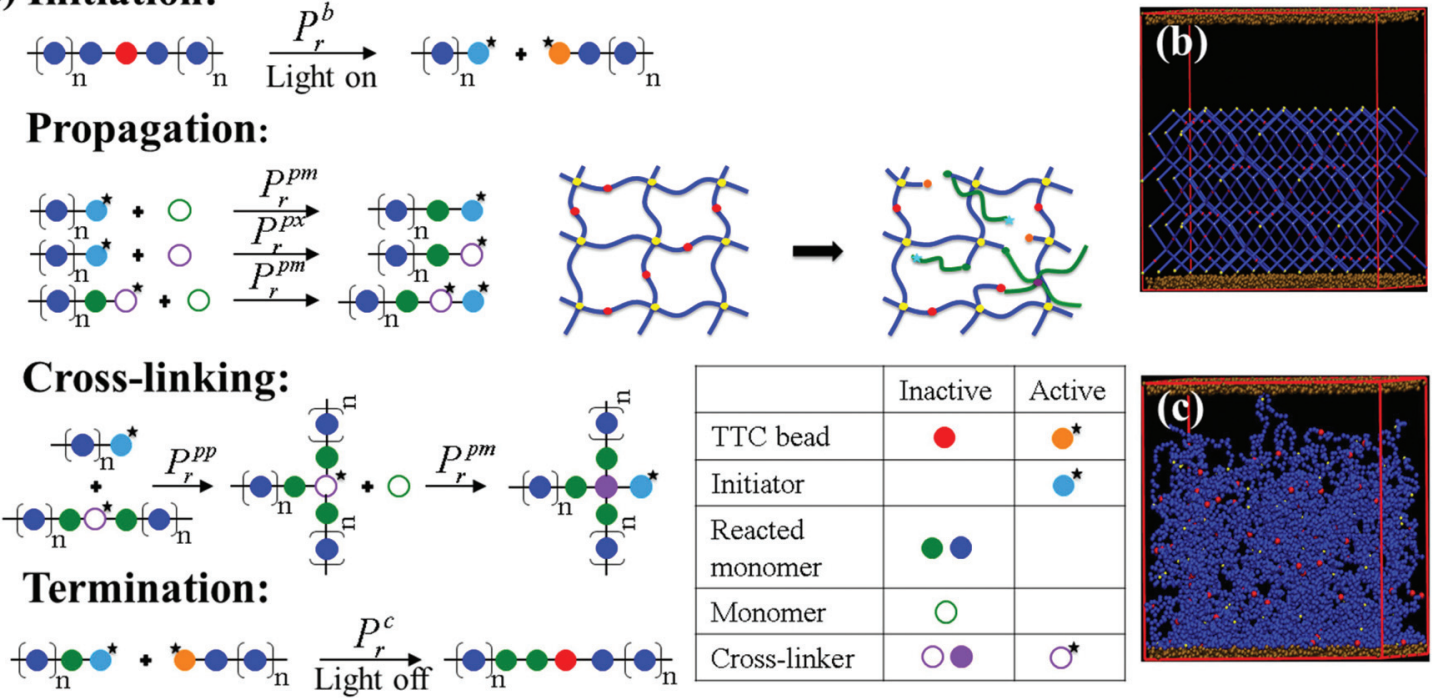

\begin{tabular}{|l|c|c|}
\hline & Inactive & Active \\
\hline TTC bead & $\ddots$ & ${ }^{\star}$ \\
\hline Initiator & & ${ }^{\star}$ \\
\hline $\begin{array}{l}\text { Reacted } \\
\text { monomer }\end{array}$ & $\ddots$ & \\
\hline Monomer & $\bigcirc$ & \\
\hline Cross-linker & $\bigcirc$ & $\bigcirc^{\star}$ \\
\hline
\end{tabular}

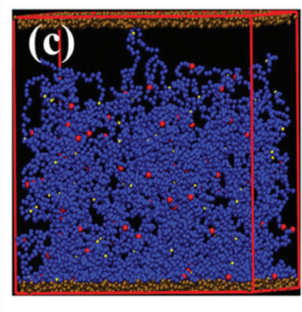

Fig. 1 (a) Schematic of the major reactions in the photo-controlled radical polymerization (photo-CRP). The details of the propagation and crosslinking steps for the related ATRP system can be found in Gao et al., ${ }^{50}$ and details for implementing the ATRP scheme within the DPD framework are given by Yong et al. ${ }^{36}$ An asterisk indicates an active radical. The following species are marked by the colors in the parentheses: inactive TTC (red), active TTC (orange), active radical (cyan), monomer (green and blue), and cross-linker within the grown gel (purple). Open beads show unreacted species and filled beads show fully reacted species. Probabilities of bond breakage $\left(P_{\mathrm{r}}^{\mathrm{b}}\right)$, propagation with the monomer $\left(P_{\mathrm{r}}^{\mathrm{pm}}\right)$, propagation with the unreacted bifunctional cross-linker $\left(P_{r}^{\text {px }}\right)$, cross-linking of partially reacted cross-linker with the pendant functional group $\left(P_{r}^{\text {pp }}\right)$, and termination $\left(P_{r}^{\mathrm{c}}\right)$ can be varied independently. (b) The initial configuration of the primary gel is a tetra-functional network with a diamond-like topology. The blue lines represent polymer strands, yellow beads are cross-linkers for the primary gel, and the red beads are TTCs. The top and bottom wall beads are shown in brown. (c) An equilibrated structure of the primary gel for $5 \times 10^{4}$ simulation time steps.

ticipate in the chain transfer reaction and hence, we can neglect this process.)

The polymerization process in our approach (specifically the propagation and cross-linking steps) is similar to that previously modeled via coarse-grained $\mathrm{MD}$ and $\mathrm{MC}$ simulations. ${ }^{49-55}$ Common to the latter methods, during each reaction step for each reactive bead, we pick at random another bead within the interaction radius $r_{i}$ of the given bead. A polymerization probability $P_{\mathrm{r}}$ is introduced to determine if the reacting pair of beads will form a bond; each successful reaction results in irreversible bond formation. The energy of the bond is given by:

$$
E_{\mathrm{b}}=1 / 2 K_{\mathrm{b}}\left(r-r_{0}\right)^{2},
$$

where $K_{\mathrm{b}}=128$ is the elastic constant and $r_{0}=0.5$ is the equilibrium bond distance.

We can modify the rate constants of the different events in the process (see Fig. 1a) ${ }^{36,37}$ by choosing different probabilities for the following reactions: photo-initiation (bond breaking) probability, $P_{\mathrm{r}}^{\mathrm{b}}$; monomer addition (propagation) probability, $P_{\mathrm{r}}^{\mathrm{pm}}$; cross-linking probabilities, $P_{\mathrm{r}}^{\mathrm{px}}$ and $P_{\mathrm{r}}^{\mathrm{pp}}$; and the termination (chain combination) probability, $P_{\mathrm{r}}^{\mathrm{c}}$. The value of $P_{\mathrm{r}}$ should, however, be sufficiently small to ensure controlled polymerization growth in the kinetically controlled reaction regime. ${ }^{55}$ For our reference case, we chose $P_{\mathrm{r}}^{\mathrm{b}}=1 \times 10^{-2}, P_{\mathrm{r}}^{\mathrm{pm}}=$ $1 \times 10^{-2}, P_{\mathrm{r}}^{\mathrm{px}}=2 P_{\mathrm{r}}^{\mathrm{pm}}$ and $P_{\mathrm{r}}^{\mathrm{c}}=2 \times 10^{-3}$. (We take the probability of termination $\left(2 \times 10^{-3}\right)$ to be smaller than the initiation probability $\left(1 \times 10^{-2}\right)$ because we assume that the sample is illuminated throughout the polymerization process and hence, the critical reactions would be the initiation, propagation and cross-linking, with few termination reactions occurring while the light is turned on.) The reaction probability $P_{\mathrm{r}}^{\mathrm{pp}}$ controlling the cross-linking process is chosen as $P_{\mathrm{r}}^{\mathrm{pp}}=3 P_{\mathrm{r}}^{\mathrm{pm}}$ to account for the fact that the bifunctional cross-linker is modeled by a single bead. ${ }^{36,37}$ We set the interaction radius to be $r_{\mathrm{i}}=0.7$ since smaller values of $r_{\mathrm{i}}$ result in delayed gelation (with respect to the corresponding experimental data) and taking $r_{\mathrm{i}}>0.7$ resulted in considerable deviations from linear firstorder kinetics. ${ }^{36,37}$ The reaction steps are separated by the reaction interval $\tau_{\mathrm{r}}$, chosen as $\tau_{\mathrm{r}}=0.2 \tau$ and thus, the reactions are performed every 10 time steps. ${ }^{52}$

The dimensions of the simulation box are $25 \times 25 \times 25$; periodic boundary conditions are imposed in the $x$ - and $y$-directions. This domain is bounded by two solid walls formed from beads that have an amorphous structure; the height of the wall is $h=1$, and the bead density is $\rho \sim 3$ (a volume fraction of $\left.\phi_{\mathrm{w}}=0.08\right)$. Bounce-back boundary conditions are applied at the fluid-solid interfaces to prevent solvent and gel beads from penetrating into the walls. These bounce-back rules also yield no-slip boundary conditions, with minimal interfacial density oscillations. $^{56}$

At the outset of the DPD simulations, we construct the primary gel as a finite-size tetra-functional network with a 
diamond-like topology, ${ }^{57}$ as shown in Fig. 1b. The polymer strands are modeled as a sequence of $N=30$ DPD beads that are connected by harmonic spring-like bonds, with an interaction potential given by eqn (1). The network has 144 crosslinks (volume fraction $\phi_{x}=3.23 \times 10^{-3}$ ) and a total of 252 strands (the chains between the cross-links). This network is composed of $N_{\text {gel }}=144+252 N$ beads (polymer volume fraction $\left.\phi_{\mathrm{p}}=0.17\right)$ and is periodic in the lateral $(x$ and $y$ ) directions. The TTCs are embedded within the centers of randomly selected polymer strands, i.e., not all the strands contain TTC beads and there is at most one TTC bead per strand. The network is attached to the bottom wall (Fig. 1b) via an adhesive interaction with the wall beads. In particular, polymer beads in the network that are in contact with the wall beads can form bonds with the wall beads. ${ }^{58}$ This adhesive interaction is modeled as a truncated Hookean spring $F_{\mathrm{adh}}=-K_{\mathrm{adh}} r_{i j}$, where $K_{\text {adh }}=10$ is the effective strength of the adhesion. These bonds break when their length exceeds $r_{\mathrm{c}}$.

A second polymer layer is grown within and on top of the primary gel, using monomers and cross-linkers that are dispersed in the solution. The initiator for the reaction is an active radical obtained from the illumination of a TTC bead. For our reference case (referred to as "case 1 " below), we set the ratio of the initial respective concentrations of initiator, cross-linker, monomer as $[\mathrm{Ini}]_{0} /[\mathrm{X}]_{0} /[\mathrm{M}]_{0}=1 / 5 / 75$ (or $\phi_{\text {Ini }}=2.14$ $\left.\times 10^{-3}, \phi_{x}=1.07 \times 10^{-2}, \phi_{m}=0.16\right)$. When the polymerization reaches full conversion, the volume fraction of the grown gel is $\phi_{\mathrm{g}}=0.17$. The volume fraction of solvent is held fixed at $\phi_{\mathrm{s}}=0.58$.

We vary the TTC concentration by selecting a strand at random that does not contain this bead and converting a polymer bead to a TTC bead. By varying the TTC concentration and fixing the volume fraction of gel, $\phi=\phi_{\mathrm{p}}+\phi_{\mathrm{g}}=0.34$ (essentially near completion of grown gel), we investigate the effect of TTC concentration on the structure of a photo-CRP gel. Namely, in addition to case 1 mentioned above, we also consider case 2 where the ratio of initial concentrations is [Ini] $]_{0} /$ $[\mathrm{X}]_{\mathrm{o}} /[\mathrm{M}]_{0}=1 / 5 / 118\left(\phi_{\text {Ini }}=1.38 \times 10^{-3}, \phi_{\mathrm{x}}=6.9 \times 10^{-3}, \phi_{\mathrm{m}}=\right.$ 0.163 ) and case 3 , where this ratio is $[\text { Ini }]_{0} /[\mathrm{X}]_{0} /[\mathrm{M}]_{0}=1 / 5 / 260$ $\left(\phi_{\text {Ini }}=6.46 \times 10^{-4}, \phi_{\mathrm{x}}=3.23 \times 10^{-3}, \phi_{\mathrm{m}}=0.167\right)$.

Within our model, variations in light intensity can be simulated by altering the probability of bond breaking $P_{\mathrm{r}}^{\mathrm{b}}$. Here, we alter $P_{\mathrm{r}}^{\mathrm{b}}$ to determine the effect of light intensity on the kinetics of the photo-CRP reaction, as well as the structure of the final gel.

The interaction parameter between the beads, $a_{i j}$, is set to $a_{i j}=25$ (in units of $k_{\mathrm{B}} T / r_{\mathrm{c}}$ ) for any two identical or chemically compatible beads. ${ }^{44}$ The value of the interaction parameter between the incompatible beads is set to $a_{i j}=60$. The primary gel, monomer and cross-linker beads are immersed in the host solvent. Initially, the monomer and cross-linker are randomly distribution in this solution and the primary gel has the configuration shown in Fig. 1b. We equilibrate the system for $5 \times 10^{4}$ simulation time steps at the specified temperature before introducing the photo-CRP reactions (see Fig. 1c).

\section{Results and discussion}

Using our newly developed DPD scheme, we first analyze the polymerization kinetics that characterize the photo-growth process by varying the probability of bond breaking $P_{\mathrm{r}}^{\mathrm{b}}$, which controls the rate of photo-initiation (see Fig. 1a). We keep all other probabilities the same as those in the reference case (shown in Fig. 2). Such variations in $P_{\mathrm{r}}^{\mathrm{b}}$ can in principle be achieved experimentally by varying the light intensity (assuming that irradiation occurs evenly throughout the gel sample). In particular, in the iniferter-based photo-CRP reaction modeled here, it is known that decreasing the light intensity decreases the molar mass dispersity likely due to a reduced concentration of propagating radicals. $^{29,32}$

We plot the monomer conversion (Fig. 2a) and $\ln \left([\mathrm{M}]_{0} /[\mathrm{M}]\right.$ ) (Fig. 2b) as a function of time (in simulation time steps) for case 1 with compatible gels $\left(a_{i j}=25\right)$, where we have decreased $P_{\mathrm{r}}^{\mathrm{b}}$ to $1 \times 10^{-3}$ (red curve) and $5 \times 10^{-4}$ (green curve). (The latter value is $1 / 20$ of the reference value of $P_{\mathrm{r}}^{\mathrm{b}}$.) Here, $[\mathrm{M}]_{\mathrm{o}} /[\mathrm{M}]$ represents the ratio of the initial monomer concentration to the concentration of unreacted monomer at a given time. Fig. 2a shows that as anticipated, the monomer conversion proceeds at a slower rate when $P_{\mathrm{r}}^{\mathrm{b}}$ is decreased. Namely, as noted above, with a lower value of $P_{\mathrm{r}}^{\mathrm{b}}$, there are fewer active ends at a given time, and hence, a reduction in the rate of incorporating monomer into a growing chain. Correspondingly, Fig. 2b shows that at given time, there is a higher concentration of unreacted monomer in the solution when $P_{\mathrm{r}}^{\mathrm{b}}$ is decreased.

The nearly linear dependence of $\ln \left(\left[\mathrm{M}_{0}\right] /[\mathrm{M}]\right)$ versus time shows that our DPD polymerization scheme accurately reproduces the pseudo first order kinetics that is characteristic of living radical polymerization, ${ }^{29,38-41}$ and is consistent with experimental findings for iniferter-based photo-CRP. ${ }^{29}$ In case 3 , the monomer conversion and $\ln \left(M_{0} / M\right)$ as a function of time are consistent with the results for case 1, as shown in Fig. 2c and $\mathrm{d}$.

\section{A. Formation of composite gels with compatible monomer}

Our aim is to examine the effect of varying the TTC concentration on the structure of a gel formed via the photo-CRP reaction when we start from a primary gel and compatible monomers and cross-linkers in solution. We set $a_{i j}=25$ for the following interaction parameters: monomers in the primary and secondary gel (blue and green beads, respectively), the monomers and solvent, and the monomers and cross-linkers. In this manner, we model the fact that the primary and secondary gels are chemically compatible and are equally compatible with the solvent. Under illumination, the TTC embedded within the primary gel fragments and creates two radicals. The reaction proceeds as described in Fig. 1a and ultimately results in the formation of a composite gel (marked in blue and green). (The different colors aid in visualizing the interface between the compatible layers.) Examples of such composite gels are shown in Fig. 3a-f. For each case, the monomer and cross-linker conversion have reached near completion 
(a)

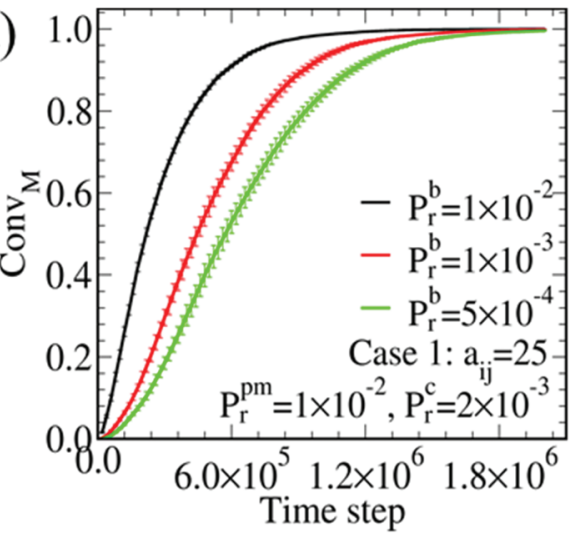

(c)

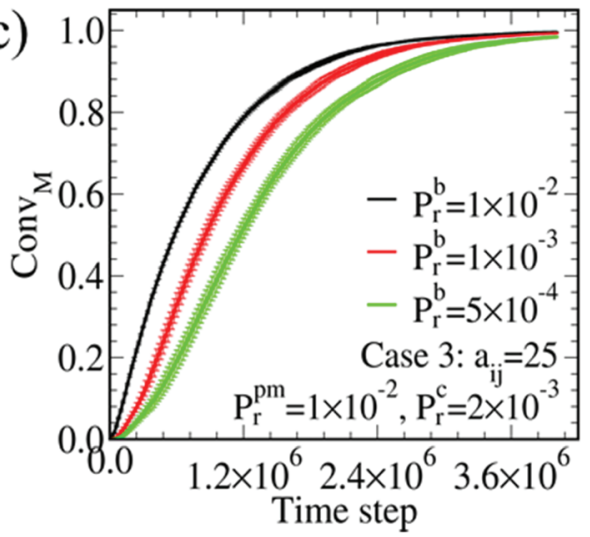

(b)

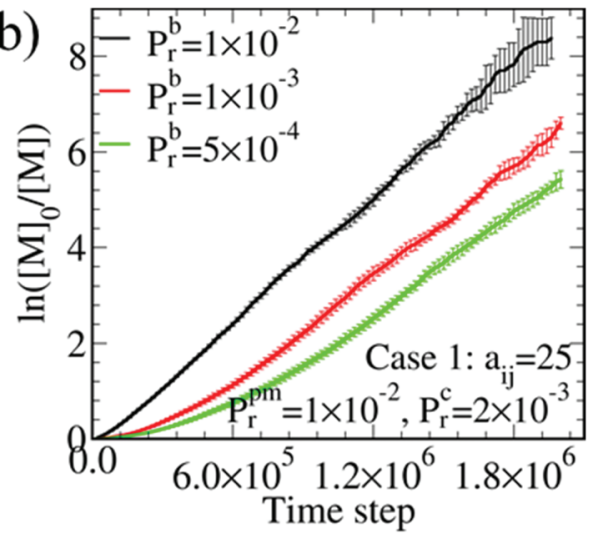

(d)

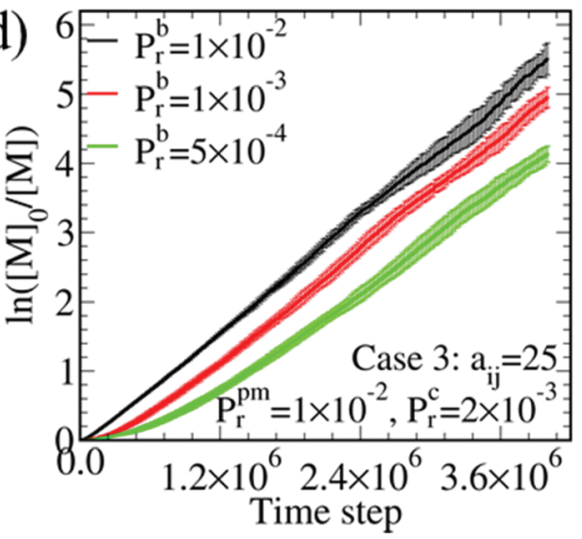

Fig. 2 The monomer conversion (in (a) and (c)) and $\ln \left([M]_{0} /[M]\right)$ (in (b) and (d)) as a function of the number of time steps during the polymerization for compatible gels $\left(a_{i j}=25\right)$, where $[M]$ is the current concentration of unreacted monomer. The dimensionless simulation time (reaction time) is the number of time steps multiplied by the time step $(\Delta t=0.02)$. (a-b) Data for case $1\left(\phi_{\text {Ini }}=2.14 \times 10^{-3}\right)$ and $(c-d)$ data for case $3\left(\phi_{\text {Ini }}=6.46 \times\right.$ $10^{-4}$ ) at different values of $P_{\mathrm{r}}^{\mathrm{b}}$ as given in legends. Error bars are from averaging over five independent runs.

(i.e., $\sim 100 \%$ ) with reaction probabilities $P_{\mathrm{r}}^{\mathrm{b}}=0.01, P_{\mathrm{r}}^{\mathrm{pm}}=0.01$ and $P_{\mathrm{r}}^{\mathrm{c}}=0.01$.

Case 1 corresponds to the highest TTC concentration in our study (Fig. 3a); here, the ratios of the TTC, monomer, and cross-linker concentrations in the solution are set as $[\mathrm{Ini}]_{\mathrm{O}} /[\mathrm{X}]_{0} /$ $[\mathrm{M}]_{0}=1 / 5 / 75$. The photo-initiated fragmentations create a large number of pores (this number is proportional to the number of TTCs) in the primary gel. The presence of these pores and the compatibility between the monomers comprising the primary (blue) and grown (green) gels facilitates the intermixing of these different species and enables the simultaneous growth of the secondary gel from multiple locations within the primary network. Fig. 3b shows the isosurface of the composite shown in Fig. 3a. The open space represents the grown gel and the interface between the primary and grown gels is shown in yellow. Fig. 3a and b clearly show that the gels in case 1 are well intermixed.

Case 2 encompasses the intermediate TTC concentration (Fig. 3c); here, the ratios of the concentrations of the various species are $[\mathrm{Ini}]_{0} /[\mathrm{X}]_{0} /[\mathrm{M}]_{0}=1 / 5 / 118$. The lower concentration of TTCs leads to fewer pores in the primary gel than in case 1. Hence, in this case, even a compatible gel grown from the primary matrix does not intermix as well with the primary gel as in case 1. Fig. 3c and d (the isosurface plot) reveal that the system shows a degree of segregation between the primary and grown gel, though the differences between cases 1 and 2 are not dramatic.

Much larger differences are observed when the concentration of the TTC embedded within the primary gel is reduced further. Namely, in case 3 , the concentration ratios are [Ini $]_{0} /$ $[\mathrm{X}]_{0} /[\mathrm{M}]_{0}=1 / 5 / 260$. Even if both species are miscible, Fig. 3e and $f$ reveal a high degree of spatial segregation between the primary and grown gel. At this low TTC concentration, the number of initiating sites leads to only a small number of pores within the bulk of the primary gel. Hence, most of the free volume available for the second gel is located on the surface of the primary network.

The spatial density distributions for both the primary and secondary gels along the transverse $(z)$ direction are plotted for each case in Fig. $3 \mathrm{~g}$ and $\mathrm{h}$. The plots indicate that at a fixed gel concentration, the degree of intermixing within the composite gel can be tailored by varying the TTC concentration. (Since the primary gel is attached to the bottom wall, the number density of this gel is highest near the wall, i.e., at $z \leq 3$.) In 


\section{Composite gel}

(a)

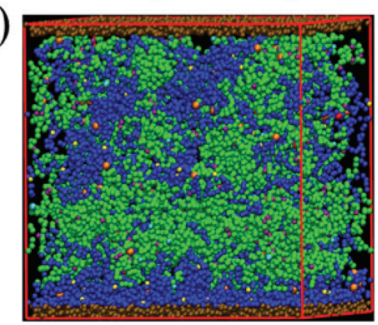

(c)

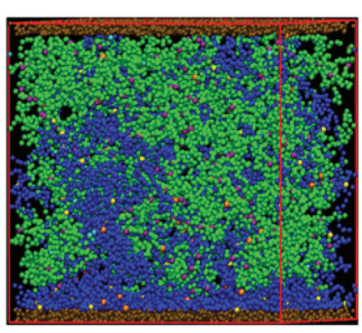

(e)

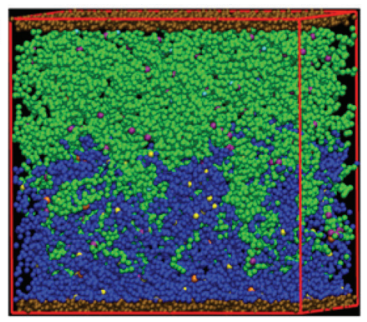

(b)

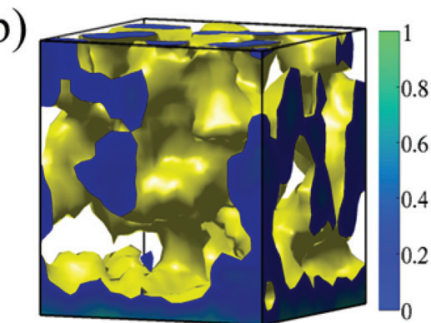

(d)

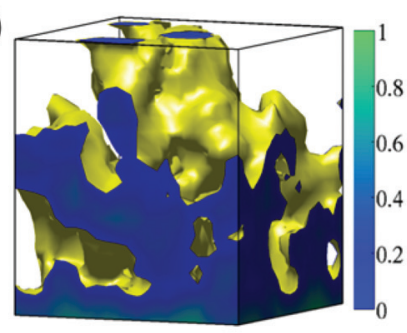

(f)

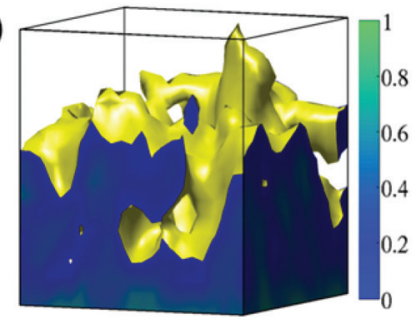

(g)

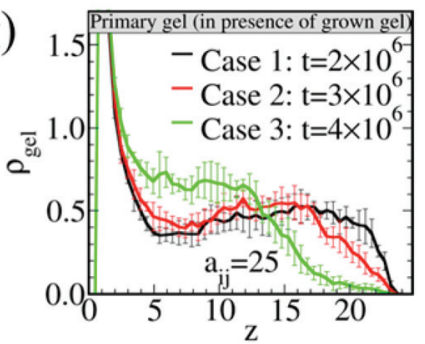

(h)

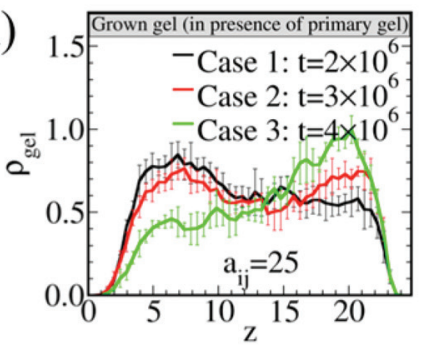

(i)

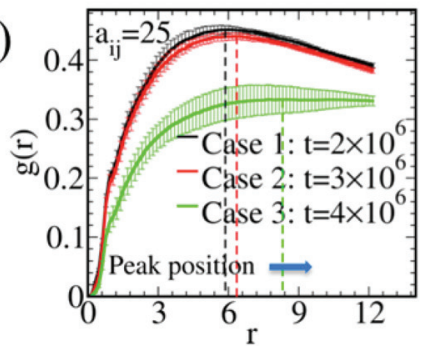

Fig. 3 Composites created by the photo-CRP approach for compatible monomers $\left(a_{i j}=25\right)$. (a) Snapshot of the composite gel at a high TTC concentration (case 1: $\phi_{\mathrm{Ini}}=2.14 \times 10^{-3}$ ). The blue and green beads represent the primary gel and the grown gel (monomer conversion: $\sim 100 \%$ ) respectively. (b) Isosurface of the composite gel shown in (a). The open space represents grown gel. The interface of the primary and grown gels is shown in yellow. (c-d) A snapshot and its respective isosurface at an intermediate TTC concentration (case 2: $\phi_{\mathrm{Ini}}=1.38 \times 10^{-3}$ ). $(\mathrm{e}-\mathrm{f}) \mathrm{A}$ snapshot and its respective isosurface at a low $T \mathrm{C}$ concentration (case 3: $\left.\phi_{\mathrm{Ini}}=6.46 \times 10^{-4}\right)$. ( $\mathrm{g}-\mathrm{h}$ ) Number density profiles of primary (in g) and grown (in h) gels for various TTC concentrations (cases 1-3). (i) Comparison of the radial distribution functions (RDF) of the composite gels. The reaction probabilities are $P_{\mathrm{r}}^{\mathrm{b}}=1 \times 10^{-2}, P_{\mathrm{r}}^{\mathrm{pm}}=1 \times 10^{-2}$ and $P_{\mathrm{r}}^{\mathrm{c}}=2 \times 10^{-3}$. The monomer conversion is $100 \%$ at time steps shown in the plots.

cases 1 and 2 (black and red curves, respectively), the density profiles of both gels are relatively uniform throughout the simulation box due to the high concentration of TTCs and resultant number of initiation sites. In case 3 (shown in green), however, the density profile of the primary gel is strongly shifted towards the bottom wall, while the secondary gel is localized towards the top wall. The latter plot confirms that the chemically miscible gels have segregated into two distinct domains.

To further characterize the spatial distribution of the green and blue beads within the gel, we also calculate the radial distribution function (RDF), $g(r)$. In particular, we measure the probability of a green bead being at a distance $r_{i j}=\left(\left|\mathrm{d} x_{i j}\right|^{2}+\right.$ $\left.\left|\mathrm{d} y_{i j}\right|^{2}+\left|\mathrm{d} z_{i j}\right|^{2}\right)^{1 / 2}$ from a given blue bead. Recall, however, that our system is periodic in the $x$ - and $y$-directions; hence, we assume that the upper bound of $\mathrm{d} x_{i j}$ and $\mathrm{d} y_{i j}$ is less than or equal to half the width of the periodic box..$^{59}$ On the other hand, the upper limit of $\mathrm{d} z_{i j}$ is the width of the box as the system is bounded by hard walls in the $z$-direction. Using these bounds in the calculation of the RDF, we effectively characterize the structure of the composite gel in the vertical direction.

In the cases involving relatively high TTC concentrations (black and red curves), the high peak of $g(r)$ at small $r$ indicates that the blue particles are closely clustered around the green beads, indicative of an intermixed system (Fig. 3i). In case 3, which involves low TTC concentration, the peak height for $g(r)$ is significantly reduced relative to the other cases and the peak position is shifted to a higher $r$. The latter features indicate that in case 3, fewer blue beads lie in proximity to the green beads and the average distance between a blue and green bead is greater than in cases 1 and 2. These features indicate that the green and blue domains are more segregated in case 3 than in the other instances.

Taken together, the data in Fig. 3 clearly show that the primary and grown gels become spatially separated as the TTC concentration is reduced. The fact that cases 1 and 2 display quite similar behavior indicates that the TTC concentration 
must be reduced below a certain critical level for this phenomenon to occur.

\section{B. Formation of composite gels with incompatible monomer}

We now examine a photo-growth system of immiscible monomers in a mutually compatible solvent and investigate the effect of varying the TTC concentration on the polymer network structure. Here, we set the interaction parameter between the different monomer beads to $a_{i j}=60$ and between the solvent and monomers to $a_{i j}=25$. The reaction probabilities are set to the values used in our reference case and the respective TTC concentrations are the same as in cases 1-3 above.

The morphologies of the composite gels and the corresponding isosurfaces for the different cases are shown in Fig. 4a-f. Remarkably, at the highest TTC concentration (case 1, Fig. 4a and b), the composite gels formed from the incompatible monomers are relatively intermixed. As noted above, at this high concentration of TTCs there are a large number of initiation sites, and hence, a significant amount of additional free volume (pores) in the system. The monomers can diffuse through the pores and then react with the photo-generated initiator. Since the initiator is a free radical, it attacks and attaches the free monomer; the energetic drive for binding the monomer to the chain overwhelms the enthalpic differences between the incompatible monomers. Hence, the blue monomer is added to the green chain. (Recall that living freeradical polymerization techniques are used to form diblock copolymers from incompatible monomers. ${ }^{60,61}$ )

The structure of the system at high TTC concentration is quite different from that at the lowest value of the TTC concentration considered here (case 3, Fig. 4c and d). In the latter case, the relatively low photo-initiated bond breakage leads to a clear phase separation between the two immiscible gels. The effects of varying the TTC concentration are also apparent from the density profiles shown in Fig. $4 \mathrm{~g}$ and $\mathrm{h}$, which highlight the pronounced phase separation between the primary and grown gels at the lowest TTC concentration.

A comparison of the number density profiles for the scenarios involving the miscible and immiscible monomers pro- (a)

\section{Composite gel}

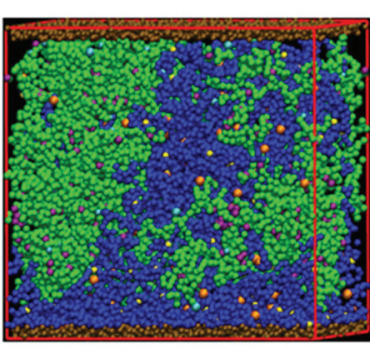

(c)

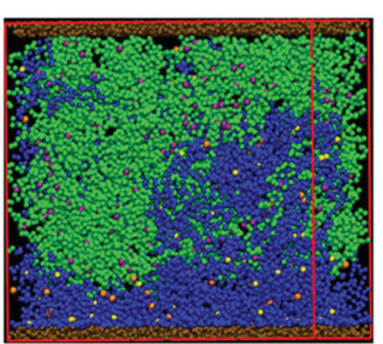

(e)

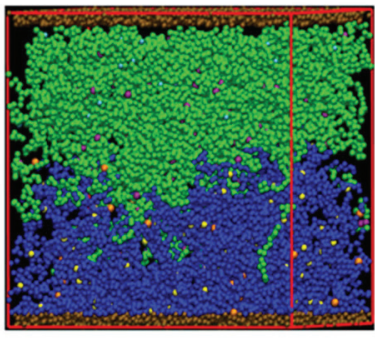

(b)

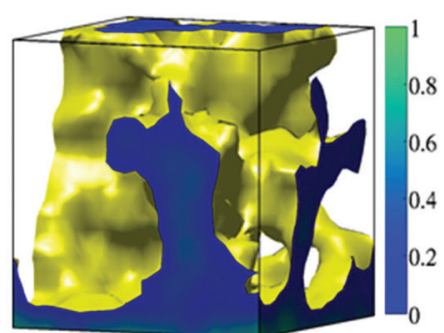

(d)

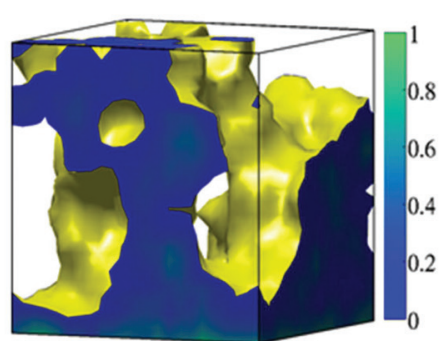

(f)

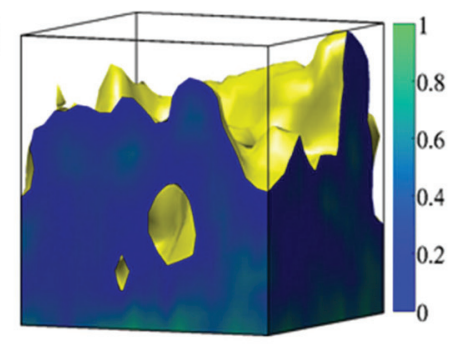

(g)

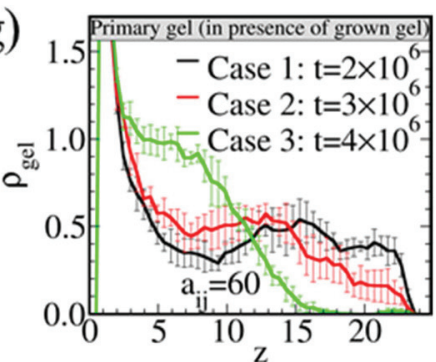

(h)

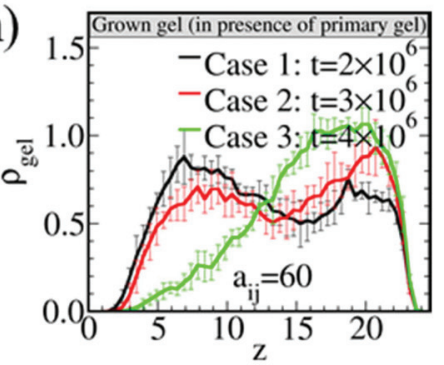

(i)

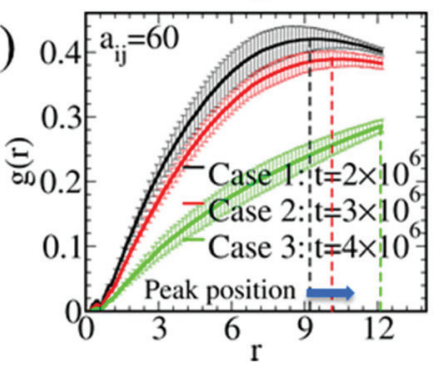

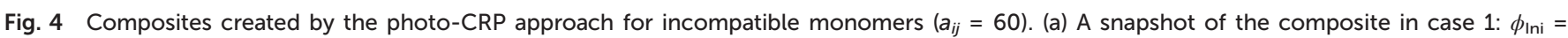

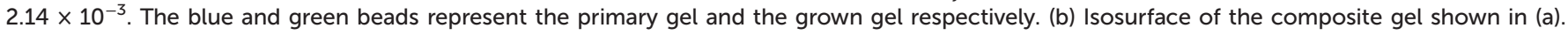

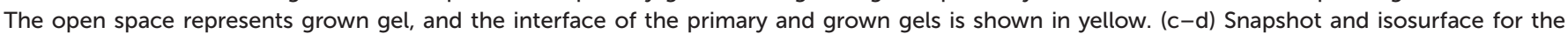

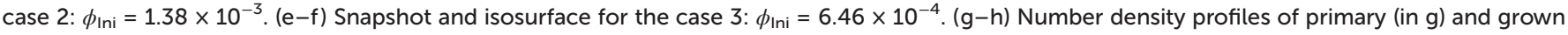

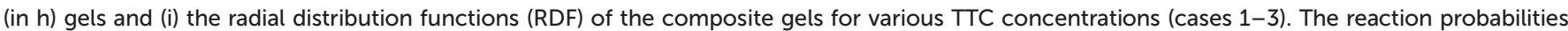
are $P_{\mathrm{r}}^{\mathrm{b}}=1 \times 10^{-2}, P_{\mathrm{r}}^{\mathrm{pm}}=1 \times 10^{-2}$ and $P_{\mathrm{r}}^{\mathrm{c}}=2 \times 10^{-3}$. The monomer conversion is $100 \%$ at time steps shown in the plots. 
(a)

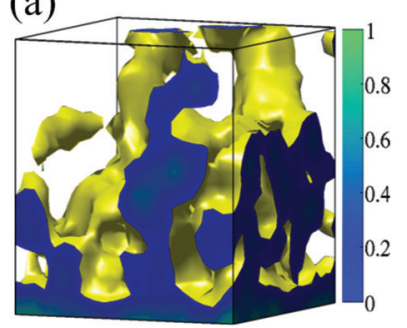

(e)

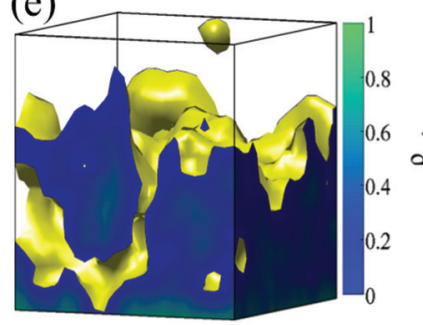

(b)

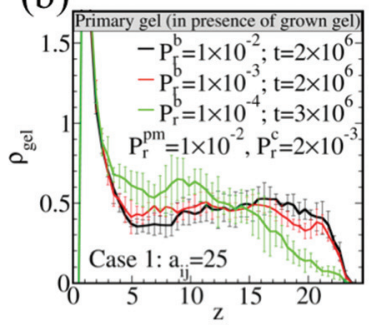

(f)

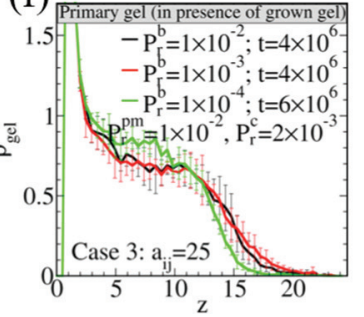

(c)

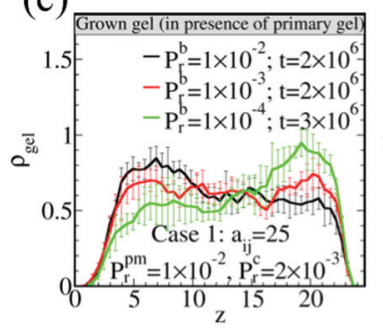

(d)

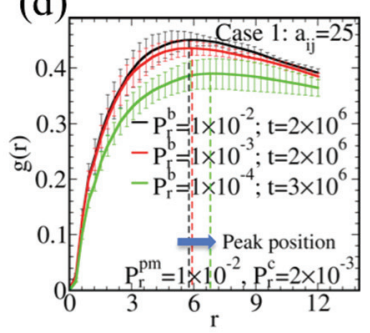

(g)

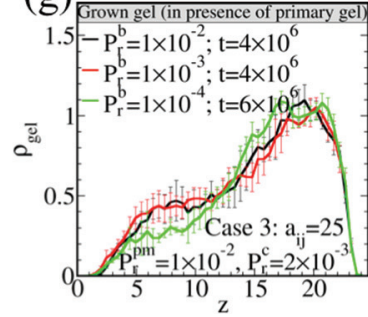

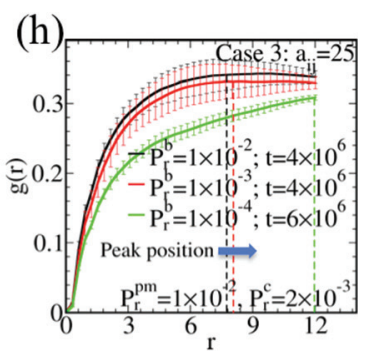

Fig. 5 Comparison of evolution snapshots (isosurfaces), number density profiles and radial distribution functions of compatible gels ( $\left.a_{i j}=25\right)$ for $(\mathrm{a}-\mathrm{d})$ case 1 and $(\mathrm{e}-\mathrm{h})$ case 3 . The volume fractions of TTCs and cross-linkers for case 1 and case 3 are $\phi_{\text {Ini }}=2.14 \times 10^{-3}, \phi_{\mathrm{x}}=1.07 \times 10^{-2}$ and $\phi_{\text {Ini }}=$ $6.46 \times 10^{-4}, \phi_{\mathrm{x}}=3.23 \times 10^{-3}$. The isosurfaces shown in (a) and (e) are at photo-intensity: $P_{\mathrm{r}}^{\mathrm{b}}=1 \times 10^{-4}$. (b-c) Number density profiles of primary (in b) and grown (in c) gels for case 1, and ( $\mathrm{f}-\mathrm{g}$ ) the same for case 3. Radial distribution functions for case 1 and case 3 are shown in (d) and (h).

vides further insight into the structure of the gel in the latter case. In particular, the number density profiles of both primarily and secondary gels are less uniform (more separated) in the incompatible monomer case (Fig. $4 \mathrm{~g}$ and $\mathrm{h}$ ) compared to the respective scenarios for the compatible monomers (Fig. $3 \mathrm{~g}$ and h). As an example, in the case 3 the incompatible gels show significantly more pronounced separation than the compatible gels.

The radial distribution functions (RDF) plotted in Fig. 4i show that the peak of $g(r)$ is reduced and shifted to the higher value of $r$ as the TTC concentration is reduced. By comparing the RDF plots across the different cases, we find the same trend when the interaction parameter between the monomers is increased from $a_{i j}=25$ to $a_{i j}=60$. This behavior is consistent with the trends seen in the snapshots and the corresponding isosurfaces shown in Fig. 3a-f and $4 \mathrm{a}-\mathrm{f}$, respectively.

\section{Effect of light intensity on the morphology of the composite gel}

As shown in Fig. 2, the light intensity $\left(P_{\mathrm{r}}^{\mathrm{b}}\right)$ has a significant impact on the time dependence of monomer conversion and $\ln \left([\mathrm{M}]_{\mathrm{o}} /[\mathrm{M}]\right)$. Based on these observations, we examine the effect of light intensity on the structural properties of the composite gels, focusing on the point where the green gel is near completion of the polymerization process, i.e., at $\sim 100 \%$ monomer conversion. Fig. 5a and e show the isosurfaces of the compatible composite gels $\left(a_{i j}=25\right)$ at $P_{\mathrm{r}}^{\mathrm{b}}=1 \times 10^{-4}$ (i.e., one-hundredth of the photo-intensity of the reference case) for case 1 and case 3, respectively. The corresponding isosurfaces of composite gels for the reference case are shown in Fig. 3b and f. In Fig. 5b, c, f and g, we compare density profiles and in Fig. $5 \mathrm{~d}$ and $\mathrm{h}$, we compare the radial distribution functions for the above two cases. The effect of varying the light intensity on the composite gel structure is not apparent for $P_{\mathrm{r}}^{\mathrm{b}}=1 \times 10^{-2}$ (black line) and $P_{\mathrm{r}}^{\mathrm{b}}=1 \times 10^{-3}$ (red line); the results are statistically similar within the error bars. Even for $P_{\mathrm{r}}^{\mathrm{b}}=5 \times 10^{-4}$ i.e., one-twentieth of the photo intensity of the reference case, the structures look similar (for the sake of clarity, the results for this value are not shown here).

For $P_{\mathrm{r}}^{\mathrm{b}}=1 \times 10^{-4}$, however, the density profiles and radial distribution function (green lines) exhibit clear signs of the formation of a segregated grown gel layer in case 1 . The reason for this behavior could be that only a few TTCs are activated at this low light intensity, and complete polymerization takes place from these few sites. Therefore, as anticipated, the morphology of the composite gel in case 1 (high concentration of TTC) with very low photo-intensity is statistically similar to the morphology in case 3 (low concentration of TTC) with high photo-intensity. We note that in experimental systems where fast degenerative chain transfer can occur, it may not be possible to segregate initiation sites in this way; even initiation of a small number of sites could lead to growth throughout the gel if the TTC concentration is high and chain transfer is fast. In case 3 , it is evident that the thickness of the segregated gel layer increased even further as we decrease the photo-intensity (Fig. 5e-h). The error bars arise from averaging over five independent runs.

\section{Conclusions}

We devised a dissipative particle dynamics (DPD) approach for modeling the formation of composite gels via a photo-growth process that uses photo-controlled radical polymerization 
(photo-CRP) based on TTC iniferters embedded within a polymer network. ${ }^{29}$ Since polymer growth occurs only in the presence of light, the system provides a distinctive means of turning the polymerization "on" and "off". ${ }^{29,47}$

Using our new DPD model, we investigated the effect of varying the light intensity on the polymerization kinetics. The monomer conversion and the value of $\ln \left(\left[\mathrm{M}_{0}\right] /[\mathrm{M}]\right)$ as a function of time decreased significantly as the light intensity was decreased. The linear behavior of $\ln \left(\left[\mathrm{M}_{0}\right] /[\mathrm{M}]\right)$ versus time confirmed that our model accurately captured the first-order kinetics of living radical polymerization.

We then focused on a primary gel immersed in a solution that contained monomer and cross-linker. We investigated the effect of varying the concentration of TTC within the primary network on the photo-CRP and the morphology of the new composite gel for both compatible and incompatible monomers. Here, we fixed the total gel concentration, i.e., the sum of the primary and grown gel concentrations. At low TTC concentration, gels formed from compatible monomers exhibited a distinct segregation into two layers. Conversely, at a high TTC concentration, incompatible monomers could form a spatially intermixed material. Hence, by tailoring the TTC concentrations, one can either mix immiscible monomers or separate miscible monomers within a single composite.

The TTC concentration controls the extent of bond fragmentation within the primary gel. At high TTC concentrations, there are a large number of initiation sites, and hence, the pores that are formed provide additional free volume within the primary gel. The presence of these pores results in the simultaneous growth of the second network from multiple locations within the first gel, and thus, leads to the intermixing between the two gels. While this intermixing is enhanced for the compatible species, it is also pronounced for the incompatible monomers. At a low TTC concentration, a much lower number of initiation sites are present, and consequently, a significantly smaller fraction of additional pores are created within the first gel. Therefore, in this scenario, the second gel grows primarily from the surface of the first. It is in this manner that the miscible monomers are segregated into distinct layers.

This ability to tune the morphology of a system formed from a given set of monomers by varying the TTC concentrations (in the primary network) provides a route to creating chemically distinct regions within gels by using incompatible components or forming layered structures from chemically identical units. Overall, our photo-growth approach provides a robust route for tuning the morphology, and hence, the potential functionality of photo-responsive gels.

\section{Acknowledgements}

ACB gratefully acknowledges financial support from the Department of Energy under DOE award DE-FG02 90ER45438. JAJ gratefully acknowledges financial support from the National Science Foundation DMREF program (CHE-1334703).

\section{References}

1 Y. Luo and S. Shoichet Molly, Nat. Mater., 2004, 3, 249-253.

2 A. Lendlein, H. Y. Jiang, O. Junger and R. Langer, Nature, 2005, 434, 879-882.

3 T. F. Scott, A. D. Schneider, W. D. Cook and C. N. Bowman, Science, 2005, 308, 1615-1617.

4 J. A. Johnson, M. G. Finn, J. T. Koberstein and N. J. Turro, Macromolecules, 2007, 40, 3589-3598.

5 J. A. Johnson, J. M. Baskin, C. R. Bertozzi, J. T. Koberstein and N. J. Turro, Chem. Commun., 2008, 3064-3066.

6 A. M. Kloxin, A. M. Kasko, C. N. Salinas and K. S. Anseth, Science, 2009, 324, 59-63.

7 Y.-L. Zhao and J. F. Stoddart, Langmuir, 2009, 25, 84428446.

8 C. A. DeForest and K. S. Anseth, Nat. Chem., 2011, 3, 925-931.

9 B. D. Fairbanks, S. P. Singh, C. N. Bowman and K. S. Anseth, Macromolecules, 2011, 44, 2444-2450.

10 M. Burnworth, L. M. Tang, J. R. Kumpfer, A. J. Duncan, F. L. Beyer, G. L. Fiore, S. J. Rowan and C. Weder, Nature, 2011, 472, 334-338.

11 Y. Amamoto, J. Kamada, H. Otsuka, A. Takahara and K. Matyjaszewski, Angew. Chem., Int. Ed., 2011, 50, 16601663.

12 C. J. Kloxin, T. F. Scott, H. Y. Park and C. N. Bowman, Adv. Mater., 2011, 23, 1977-1981.

13 Y. Amamoto, H. Otsuka, A. Takahara and K. Matyjaszewski, ACS Macro Lett., 2012, 1, 478-481.

14 H. Zhao, E. S. Sterner, E. B. Coughlin and P. Theato, Macromolecules, 2012, 45, 1723-1736.

15 J. Kim, J. A. Hanna, M. Byun, C. D. Santangelo and R. C. Hayward, Science, 2012, 335, 1201-1205.

16 M. B. Gordon, J. M. French, N. J. Wagner and C. J. Kloxin, Adv. Mater., 2015, 27, 8007-8010.

17 M. Tanabe, G. W. M. Vandermeulen, W. Y. Chan, P. W. Cyr, L. Vanderark, D. A. Rider and I. Manners, Nat. Mater., 2006, 5, 467-470.

18 S. Yamago, Y. Ukai, A. Matsumoto and Y. Nakamura, J. Am. Chem. Soc., 2009, 131, 2100-2101.

19 B. P. Fors and C. J. Hawker, Angew. Chem., Int. Ed., 2012, 51, 8850-8853.

20 D. Konkolewicz, K. Schroder, J. Buback, S. Bernhard and K. Matyjaszewski, ACS Macro Lett., 2012, 1, 1219-1223.

21 N. J. Treat, H. Sprafke, J. W. Kramer, P. G. Clark, B. E. Barton, J. R. de Alaniz, B. P. Fors and C. J. Hawker, J. Am. Chem. Soc., 2014, 136, 16096-16101.

22 A. Anastasaki, V. Nikolaou, Q. Zhang, J. Burns, S. R. Samanta, C. Waldron, A. J. Haddleton, R. McHale, D. Fox, V. Percec, P. Wilson and D. M. Haddleton, J. Am. Chem. Soc., 2014, 136, 1141-1149.

23 J. T. Xu, K. Jung, A. Atme, S. Shanmugam and C. Boyer, J. Am. Chem. Soc., 2014, 136, 5508-5519.

24 K. A. Ogawa, A. E. Goetz and A. J. Boydston, J. Am. Chem. Soc., 2015, 137, 1400-1403.

25 M. Chen and J. A. Johnson, Chem. Commun., 2015, 51, 6742-6745. 
26 F. A. Leibfarth, K. M. Mattson, B. P. Fors, H. A. Collins and C. J. Hawker, Angew. Chem., Int. Ed., 2013, 52, 199-210.

27 J. T. Trotta and B. P. Fors, Synlett, 2016, 702-713.

28 M. Chen, M. Zhong and J. A. Johnson, Chem. Rev., 2016, DOI: $10.1021 /$ acs.chemrev.5b00671.

29 H. X. Zhou and J. A. Johnson, Angew. Chem., Int. Ed., 2013, 52, 2235-2238.

30 T. Otsu, M. Yoshida and T. Tazaki, Makromol. Chem., Rapid Commun., 1982, 3, 133-140.

31 A. R. Kannurpatti, S. X. Lu, G. M. Bunker and C. N. Bowman, Macromolecules, 1996, 29, 7310-7315.

32 M. Chen, M. J. MacLeod and J. A. Johnson, ACS Macro Lett., 2015, 4, 566-569.

33 S. Shanmugam, J. T. Xu and C. Boyer, Angew. Chem., Int. Ed., 2016, 55, 1036-1040.

34 K. Jung, J. T. Xu, P. B. Zetterlund and C. Boyer, ACS Macro Lett., 2015, 4, 1139-1143.

35 J. Yeow, J. T. Xu and C. Boyer, ACS Macro Lett., 2015, 4, 984990.

36 X. Yong, O. Kuksenok, K. Matyjaszewski and A. C. Balazs, Nano Lett., 2013, 13, 6269-6274.

37 X. Yong, A. Simakova, S. Averick, J. Gutierrez, O. Kuksenok, A. C. Balazs and K. Matyjaszewski, Macromolecules, 2015, 48, 1169-1178.

38 K. Matyjaszewski, Macromolecules, 2012, 45, 4015-4039.

39 K. Matyjaszewski and N. V. Tsarevsky, J. Am. Chem. Soc., 2014, 136, 6513-6533.

40 K. Matyjaszewski and J. H. Xia, Chem. Rev., 2001, 101, 2921-2990.

41 J. S. Wang and K. Matyjaszewski, J. Am. Chem. Soc., 1995, 117, 5614-5615.

42 X. Yong, O. Kuksenok and A. C. Balazs, Polymer, 2015, 72, 217-225.

43 P. Espanol and P. Warren, Europhys. Lett., 1995, 30, 191196.
44 R. D. Groot and P. B. Warren, J. Chem. Phys., 1997, 107, 4423-4435.

45 P. J. Hoogerbrugge and J. M. V. A. Koelman, Europhys. Lett., 1992, 19, 155-160.

46 S. Plimpton, J. Comput. Phys., 1995, 117, 1-19.

47 T. Otsu, J. Polym. Sci., Polym. Chem. Ed., 2000, 38, 21212136.

48 H. Wang, Q. B. Li, J. W. Dai, F. F. Du, H. T. Zheng and R. K. Bai, Macromolecules, 2013, 46, 2576-2582.

49 R. L. C. Akkermans, S. Toxvaerd and W. J. Briels, J. Chem. Phys., 1998, 109, 2929-2940.

50 H. F. Gao, P. Polanowski and K. Matyjaszewski, Macromolecules, 2009, 42, 5925-5932.

51 J. Genzer, Macromolecules, 2006, 39, 7157-7169.

52 H. Liu, M. Li, Z. Y. Lu, Z. G. Zhang and C. C. Sun, Macromolecules, 2009, 42, 2863-2872.

53 A. Milchev, J. P. Wittmer and D. P. Landau, J. Chem. Phys., 2000, 112, 1606-1615.

54 P. Polanowski, J. K. Jeszka and K. Matyjaszewski, Polymer, 2010, 51, 6084-6092.

55 S. Turgman-Cohen and J. Genzer, Macromolecules, 2010, 43, 9567-9577.

56 D. A. Fedosov, I. V. Pivkin and G. E. Karniadakis, J. Comput. Phys., 2008, 227, 2540-2559.

57 P. K. Jha, J. W. Zwanikken, F. A. Detcheverry, J. J. de Pablo and M. O. de la Cruz, Soft Matter, 2011, 7, 5965-5975.

58 I. Salib, X. Yong, E. J. Crabb, N. M. Moellers, G. T. McFarlin, O. Kuksenok and A. C. Balazs, ACS Nano, 2013, 7, 1224-1238.

59 B. G. Levine, J. E. Stone and A. Kohlmeyer, J. Comput. Phys., 2011, 230, 3556-3569.

60 D. A. Shipp, J. L. Wang and K. Matyjaszewski, Macromolecules, 1998, 31, 8005-8008.

61 K. Matyjaszewski and N. V. Tsarevsky, Nat. Chem., 2009, 1, 276-288. 